\title{
Knocking heads with viscosity: new insights into the bioenergetics of sperm motility
}

\author{
David F. Albertini
}

Published online: 15 April 2011

(C) Springer Science+Business Media, LLC 2011

Among the marvels of human reproduction, au naturel, is how a spermatozoan negotiates the perilous journey through the female reproductive tract. While a small and select number of sperm may populate the ampulla of the Fallopian tube, the chosen ONE - at the expense of its compatriotswill be distinguished by having confronted and overcome that last series of barriers before fusing with the oocyte plasma membrane. Having the "right stuff" to make this happen has been within the purview of spermatologists and the few who dare to venture into the wonderful world of reproductive tract secretions and the like. But only recently have advances been made that begin to uncover the unique properties of sperm, which empower them with the ability to withstand and progress through the global climate change that anticipates fertilization on a cellular scale.

This issue of JARG brings to the forefront two features of the process of fertilization that have been lost within the PubMed archives. An article by RHF Hunter and colleagues revisits the problem of the female reproductive tract microenvironment from a comparative perspective, reminding us that the challenges imposed on human sperm are not unlike those faced by other species that have made the evolutionary commitment to internal fertilization. This paper reveals a biophysical dimension of environmental flux involving changes in viscosity, viscoelasticity and temperature, as sperm transit from one microenvironment to the next. One cannot help but conclude that along this not-

Capsule Contextualizing metabolic specializations in sperm will aid in the identification and management of the causes of male infertility.

D. F. Albertini $(\bowtie)$

University of Kansas Medical Center,

Kansas City, KS 66103, USA

e-mail: dalbertini@kumc.edu so-merry sojourn, sperm must be capable of shifting gears in accordance to the kinds of environmental cues encountered - a more salutatory motility behavior that is in sharp contrast to the relatively mild selection steps taken during sperm preparation for IVF or ICSI.

Continuing along the theme of nature versus ARTdependent nurture, Dale and DeFelice (Polspermy prevention: facts and artifacts) extend such a comparative viewpoint a bit closer to the subject at hand: fertilization. In challenging conventional notions regarding the fast block to polyspermy, in particular, they emphasize that almost all of the knowledge we have for the mechanisms underlying monospermic penetration derives from studies on animal models that bear little resemblance to the human condition. Moreover, considering what has evolved in the practice of human ARTs, where virtually all natural interfaces that would be encountered by a fertilizing sperm have been eliminated (cumulus, zona), they posit that, where fertilization should be occurring on the human oocyte has become a crap shoot! That is, if there is a preferred and optimal place for sperm entry and egg activation, this is surely not the case when the ICSI pipette is aimed at anywhere but the site of the meiotic spindle. Food for thought, for sure, and an idea that is not likely to gain support until the experiments can be performed on human material.

Both of these articles bring our readership up to date at a time when questions about the long-term consequences of ICSI continue to be raised and the recurring ballad of how to choose the best embryo seems not to wane. In the end, the imperative to understand and more effectively treat male infertility is loud and clear: Motility parameters are readily monitored in the andrology lab and seeking explanations for poor motility, at a genetic and physiological level, will hopefully bring our diagnostic and treatment strategies to a 
heightened level. To this end, I would like to draw your attention to an example of a basic science breakthrough that impacts our understanding of male infertility and offers new avenues for a male contraceptive development.

A recent study published by Jimenez and coworkers (2011, PNAS 108:644-649; www.pnas.org/cgi/doi/10.1073/ pnas.1016902108) identifies a novel isoform of the Na,KATPase (ATP1A4) that is essential for male fertility in mice. In addition to their infertility, the study goes on to show that sperm from animals lacking this isoform fail when used in IVF, and exhibit decreased motility - as expected, given the essential role of this plasma membrane transporter in ion transport. This same group has extended its studies to show that, by inserting a copy of the rat gene for the ATP1A4 isoform into the mouse genome, the mouse sperm exhibited enhanced total and progressive motility (Jimenez et al., 2011, Biol. Reprod. 84:153-161 DOI 10.1095/biolreprod.110.087064). Strikingly, analysis of the re-engineered sperm showed that the hyperactivated motility typical of sperm capacitation is also increased in both total and progressive motility.

The significance of studies like those of Jimenez et al. is that they bring our understanding of sperm physiology more closely in line with the complexities of motility that are required to achieve successful fertilization. As the next decade of human ARTs continues to unfold, we look forward to discoveries like this, to guide our efforts in the diagnosis and treatment of male infertility. 